\title{
Eddy Current Losses in Cylindrical Arc Furnace Shells
}

\section{Peter F. Ryff}

Ryerson University

digital.library.ryerson.ca/object/47

Please Cite:

Ryff, P. F. (1981). Eddy current losses in cylindrical arc furnace shells. IEEE

Transactions on Industry Applications, IA-17(3), 329-333.

doi:10.1109/TIA.1981.4503948

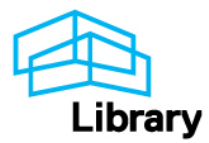




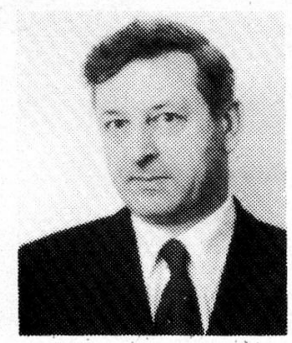

Paul G. Heyda received the B.Sc. degree from London University, London, England in 1952. Since then, he has worked as a mathematician in industry. He came to BICC Research and Engineering Ltd., London, in 1960 and is presently Head of the Theoretical Mechanics Section of the Mathematics Department.

Mr. Heyda is a Fellow of the Institute of Mathematics and its Applications.

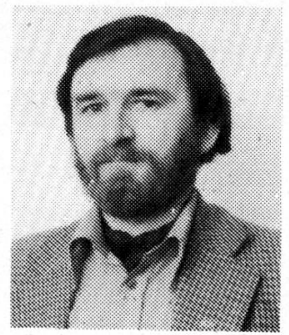

Gordon J. Thackray received the B.Sc. degree in theoretical physics and the Ph.D. degree from the University of Kent, Canterbury, England, in 1970 and 1976, respectively.

He joined the Mathematics Department of BICC Research and Engineering Ltd., London, England, in 1977 and has been involved in work on various aspects of the cable industry.

$\mathrm{He}$ is a Member of the Institute of Physics and an Associate Fellow of the Institute of Mathematics and its Applications.

\title{
Eddy Current Losses in Cylindrical Arc Furnace Shells
}

\author{
PETER F. RYFF, SENIOR MEMBER, IEEE
}

\begin{abstract}
A method is presented to determine the eddy current power losses in the shell of a three-phase cylindrical arc furnace. The effect of electrode phase rotation with unbalanced electrode currents on the induced losses is shown. This study indicates the direct similarity between the induced loss distribution and the nonlinear refractory wear pattern in a furnace. Experimental results on a furnace model are included to support the theoretical work. The measured wear pattern of a section of the refractory lining in a furnace is included to illustrate the corresponding qualitative distributions.
\end{abstract}

\section{INTRODUCTION}

B ECAUSE of substantial increases in power input with the development of ultra high power (UHP) electric arc furnaces, some of the inherited classic arc furnace operating problems such as electromagnetically induced heat losses and thermal stresses in the refractory lining are also upgraded. New developments in the arc furnace industry are constantly made to combat these problems, in view of the importance of refractory life. Understanding the phenomena is important with regard to lining wear, which is a particular major component in the economy of UHP furnaces. In extending lining wear, even moderate amounts of heat can represent a considerable saving in furnace operation. One of the latest developments is the application of water-cooled panels installed in the refractory wall above the slag line. With this innovation refractory life is greatly extended. Panel life is now

Paper IUSD 80-10, approved by the Electrostatic Processes Committee of the Industry Applications Society for publication in this TRANSACTIONS. Manuscript released for publication May 5, 1980.

The author is with Ryerson Poly technical Institute, 50 Gould St., Toronto, ON, Canada M5B 1E8. measured in the thousands of heats instead of hundreds, which is more common for refractory brick [1]. With the application of water-cooled panels and greatly increased electrode currents (80-100 kA becoming standard) in UHP furnaces, an investigation was made to determine the induced power losses in the furnace shell and forces exerted on the panels, where used. Due to the electrode arrangement being supplied with three-phase power in an electric arc furnace, as schematically illustrated in Fig. 1, a revolving magnetic field component is set up. This field will penetrate structural members that are electrically conductive and induce eddy currents with subsequent ohmic losses. Due to its revolving nature, it will exert a torque or peripheral force on those members, similar to the induction motor principle. The torque so created will be in the direction of the magnetic field. The initial investigation centered on ascertaining the magnitude of this force on the water-cooled panels with reference to the minimum mechanical securing force required. It was felt that due to the nature of the arc in the furnace, the exerted impulse forces on the panels may well become a factor to be considered.

Subsequent to the force calculations, obtained from the eddy current losses, experimental data are presented to verify the theoretical model. The effect of electrode current unbalance on the induced losses is then shown. From this it became evident, especially in view of related work carried out [2], [3], that the direction of the arcs in the furnace is influenced by this current unbalance. This of course is not entirely surprising, since an arc constitutes a "flexible" conductor on which electromagnetic forces can be exerted. However, it is interesting that the calculated eddy current loss distribution in the shell of a furnace coincides with the meas- 


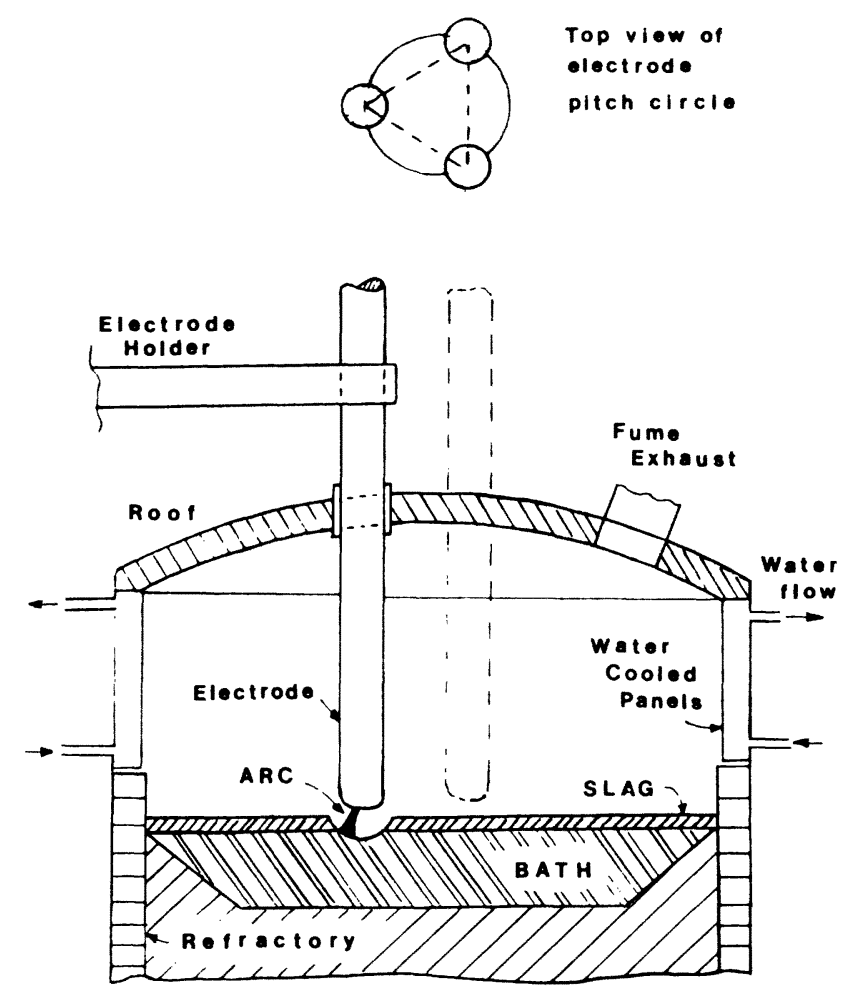

Fig. 1. Electric arc furnace with water-cooled panels.

ured nonlinear refractory wear in that same furnace to the extent that the apparent shift of the hot spot locations are identical.

\section{THEORY}

The electric arc furnace may be represented as a cylindrical shell having a radius $a$ containing the three electrodes as illustrated in Fig. 2. When water-cooled panels are installed above the slag line but covering the entire circumference, they also form a shell. The electrodes are located on the corners of an equilateral triangle, circumscribed by the electrode pitch circle of radius $b$. Because of the prevailing symmetry, cylindrical coordinates are used and the superposition theorem applied to the fields. This means that in the derivation for the vector potential in Regions 1 and 2, only one electrode (say $A$ ) is considered. The resulting expression is then extended to include electrodes $B$ and $C$, considering their proper space location and electrical phase sequence.

In the derivation it is assumed that the shell thickness is such that the field is contained within it. This assumption closely resembles practical experience because of the heavy shell. Therefore, the space occupied by the furnace can be thought of as two regions. In Region 1, the air space with permeability $\mu_{0}=4 \pi \times 10^{-7} \mathrm{H} / \mathrm{m}$, the differential equation describing the vector potential $\vec{A}_{1}$ is

$$
\nabla^{2} \bar{A}_{1}=0
$$

The differential equation describing the vector potential $\overline{A_{2}}$ in Region 2, the shell material with permeability $\mu=\mu_{0} \mu_{r}$ $\left(\mu_{r}\right.$ being the relative permeability) is

$$
\nabla^{2} \bar{A}_{2}=k^{2} A_{2}
$$

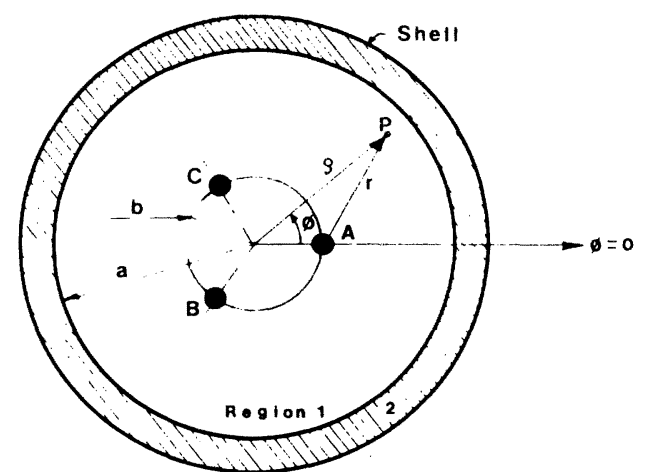

Fig. 2. Simplified arrangement of three-phase arc furnace with carbon steel shell.

with $k^{2}=i \omega \mu \sigma$, being a complex quantity. $\omega$ is the radial frequency of the supply, $i=\sqrt{-1}$ and $\sigma$ the conductivity of the shell material. The solution of (1) is given by the series [4]

$$
\bar{A}_{1}=\frac{\mu_{0} I}{2 \pi} \ln r+\mu_{0} \sum_{n=1}^{\infty} B_{n} \rho^{n} \cos n \phi
$$

where the first term is the potential due to the electrode carrying a current $I$, in which

$$
r=\left(\rho^{2}+b^{2}-2 b \rho \cos \phi\right)^{1 / 2}
$$

and represents the radial distance from the electrode axis to the point $P(\rho, \phi)$ where the field is calculated. The second term, which must be finite at the origin (positive exponent of $n$ only) is due to the magnetization of the shell material. $B_{n}$ is a constant yet to be determined. The solution of (2) may be taken of the form

$$
\bar{A}_{2}=\mu \sum_{n=1}^{\infty} D_{n} K_{n}(k \rho) \cos n \phi
$$

which must vanish at $\rho=\infty$. $K_{n}$ is the modified Bessel function of order $n$ and argument $k \rho$. $D_{n}$ is the second constant to be determined. To evaluate the unknown constants $B_{n}$ and $D_{n}$, the boundary problem on the shell's inner surface $(\rho=a)$ is solved. Here the known equations for the refraction of lines of force holds. The tangential component of the magnetic field is continuous and given by

$$
H_{1 \phi}=H_{2 \phi}, \quad \rho=a
$$

and the normal component of the magnetic flux density is also continuous and must satisfy the condition

$$
\mu_{0} H_{1 \rho}=\mu H_{2 \rho}, \quad \rho=a .
$$

Carrying out the called for derivations, equating components for each value of $n$ and realizing that

$$
H_{\phi}=-\frac{1}{\mu} \frac{\partial A}{\partial \rho}, \quad \rho=a
$$


and

$$
\frac{a b \sin \phi}{r^{2}}=\sum_{n=1}^{\infty}\left(\frac{b}{a}\right)^{n} \sin n \phi
$$

the following two equations (after making some simplifications) result, namely

$$
\frac{I}{2 \pi}\left(\frac{b}{a}\right)^{n}+n B_{n} a^{n}=D_{n} k a K_{n}^{\prime}
$$

and

$$
\frac{\mu_{0} I}{2 \pi}\left(\frac{b}{a}\right)^{n}-n \mu_{0} B_{n} a^{n}=-n \mu D_{n} K_{n}
$$

where the Bessel functions have the argument $k a$ and the prime indicates its derivative. Solving (7) and (8) simultaneously for $B_{n}$ and $D_{n}$ and using the following recurrence formula for the modified Bessel function [5], namely

$$
-K_{n}^{\prime}=K_{n+1}+\frac{n}{k a} K_{n}
$$

which will eliminate the derivative term in the final expression; the constants $B_{n}$ and $D_{n}$ are then determined.

Substituting the result for $D_{n}$ into (4) yields

$$
\bar{A}_{2}=-\frac{\mu I}{\pi} \sum_{n=1}^{\infty}\left(\frac{b}{a}\right)^{n} \frac{K_{n}(k \rho)}{\psi} \cos n \phi
$$

where

$$
\psi=k a K_{n+1}(k a)+n\left(\mu_{r}-1\right) K_{n}(k a) .
$$

Thus the fields are determined in both regions although the expression for the field in Region 2 only is of immediate interest here. Equation (9) can now be applied to the threephase arrangement as shown in Fig. 2 by applying the superposition theorem and extending the above analysis. Assuming a balanced three-phase supply of $R S T$ sequence supplied to electrodes $A, B$, and $C$, respectively, and noting that in the direction of $\Phi$, electrode $A$ is 120 degrees ahead of electrode $B$ but behind electrode $C$ by that same angle, the vector potential at any point within the shell due to all three electrodes becomes

$$
\begin{aligned}
\bar{A}_{2}= & -\frac{\mu I}{\pi} \sum_{n=1}^{\infty}\left(\frac{b}{a}\right)^{n} \frac{K_{n}(k \rho)}{\psi}\left[\cos n \phi+\alpha^{2} \cos n\left(\phi+120^{\circ}\right)\right. \\
& \left.+\alpha \cos n\left(\phi-120^{\circ}\right)\right]
\end{aligned}
$$

where the operator $\alpha$ satisfies the relations

$$
\begin{gathered}
\alpha=e^{i 2 \pi / 3}=-1 / 2+i \frac{\sqrt{3}}{2} \\
\alpha^{2}=e^{i 4 \pi / 3}=-1 / 2-\frac{i \sqrt{3}}{2}
\end{gathered}
$$

and

$$
1+\alpha+\alpha^{2}=0
$$

For an unbalanced supply current, the operators are simply altered to comply with the unbalance in question. In addition, each term must now be multiplied by its respective electrode current, since the current $I$ is different for each electrode. Finally, the desired quantity, namely, the power loss per unit volume $\left(P_{v}\right)$ at any point throughout the shell material, can now be determined from

$$
P_{v}=\sigma \bar{E}^{2}=\sigma|-i \omega \bar{A}|^{2}=\sigma \omega|\bar{A}|^{2}
$$

where $|\bar{A}|$ is the magnitude of the magnetic vector potential as determined by (10).

\section{THEORETICAL RESULTS-FURNACE MODEL}

Using the method outlined, the induced voltages were calculated in test coils assumed to be located at 20 degree intervals along the shell perimeter of a model furnace, having the dimensions illustrated in Fig. 3. The test points shown in this figure are located just outside the shell, i.e., at $\rho=\mathrm{a}$, to assimilate the physical situation where test coils are placed on its surface. Hence, they would measure voltages which are proportional to the magnetic field in Region 1 at these locations. The voltages were calculated from

$$
E=4.44 f N B_{\text {max }} A
$$

where $A$ represents the area of the test coil having $N$ number of turns. $B_{\max }$ is the magnitude of the magnetic flux density $\bar{B}$, perpendicular to the coil cross section. In other words, it is the normal component of $\bar{B}$ at the position in question, identical to that implied by (6) and obtained from

$$
B(\rho, \phi)=\frac{\partial A_{1}}{\partial \phi}, \quad \rho=a .
$$

In the computer calculations the additional needed data were taken as

$$
\begin{aligned}
& \text { shell material: } \mu_{r}=10, \sigma=5 \times 10^{6}, \\
& \text { test coil: } A=1 \text { in } \times 4 \mathrm{in}, N=25, \\
& \text { balanced three-phase supply: } I=20 \mathrm{~A} \text {, } \\
& \text { frequency: } 60 \mathrm{~Hz} .
\end{aligned}
$$

Results of these calculations are given in Fig. 4 .

\section{EXPERIMENTAL RESULTS-FURNACE MODEL}

To verify the theoretical predicted voltages, an experimental model was built according to the dimensions indicated in Fig. 3. The shell material constants were estimated and given above. Test coils were wound having the area and number of turns used in the calculations. With the electrode currents held at $20 \mathrm{~A}$, the voltages were measured with an oscilloscope and are also represented in Fig. 4. As can be seen, the agreement is excellent. The discrepancies that do exist are expected to be caused by inaccuracies in determining the material constants of the shell material and the assumption in 


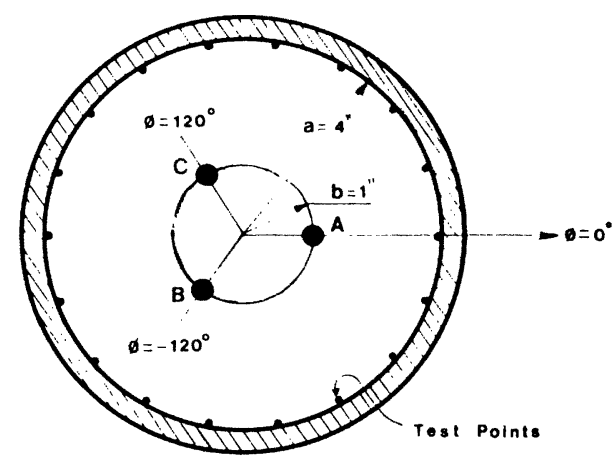

Fig. 3. Furnace model indicating dimensions and test coil locations.

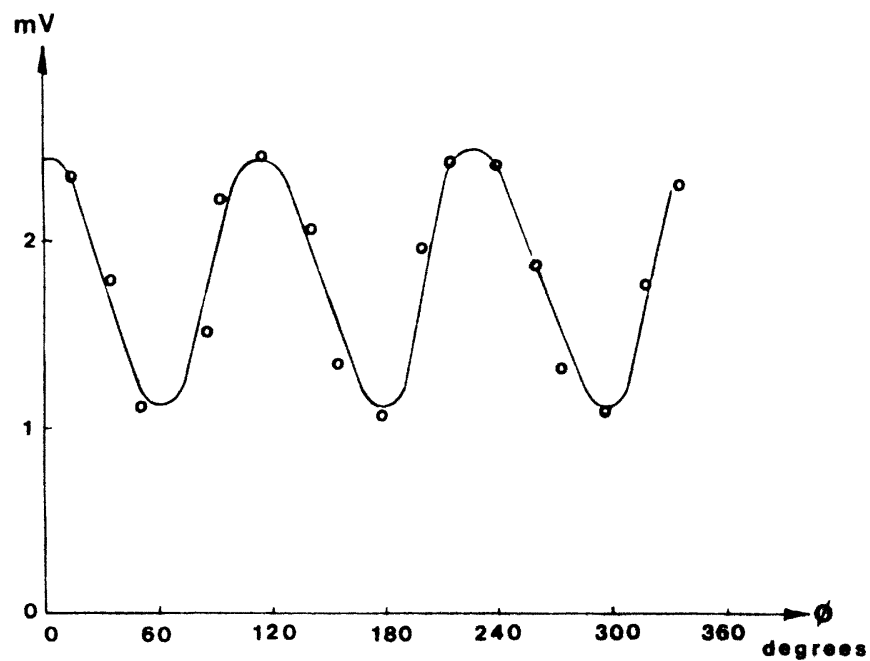

Fig. 4. Induced voltages in test coils placed as shown in Fig. 3. Solid lines are theoretical results, circles indicate experimental values.

the calculations, where the field is taken constant over the coil cross section.

\section{THEORETICAL RESULTS-ARC FURNACE}

The power losses were calculated in the shell of an electric arc furnace and the results, for the power densities along the surface, represented in Fig. 5. The specific furnace parameters are

shell diameter: $23 \mathrm{ft}, 6$ in,

electrode pitch circle diameter: $5 \mathrm{ft}, 6 \mathrm{in}$,

electrode current: $60 \mathrm{kA}$.

frequency: $60 \mathrm{~Hz}$,

electrode diameter: 24 in.

The shell material (low carbon steel) constants were taken as $\sigma=5.6 \times 10^{6}$ and $\mu_{r}=100$. It was assumed that all three phases and subsequently all three arcs perform identically, resulting in a symmetrical power loss density distribution along the circumference of the shell. However, it is wellknown in practice that one phase melts faster than another and therefore radiates more heat onto the sidewall refractory. This unbalance, caused by the differences in the ballasts of the three phases, is due mainly to variations in secondary circuit impedances. Although efforts are made to rectify this by transposing the heavy current conductors in an equilateral triangle, this desired condition can seldom be maintained

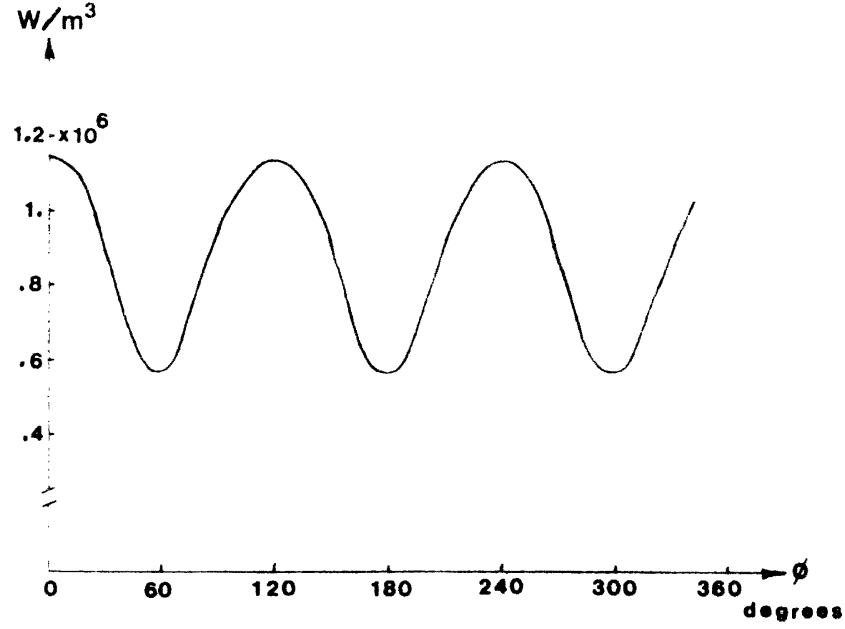

Fig. 5. Power loss density along surface of furnace shell.

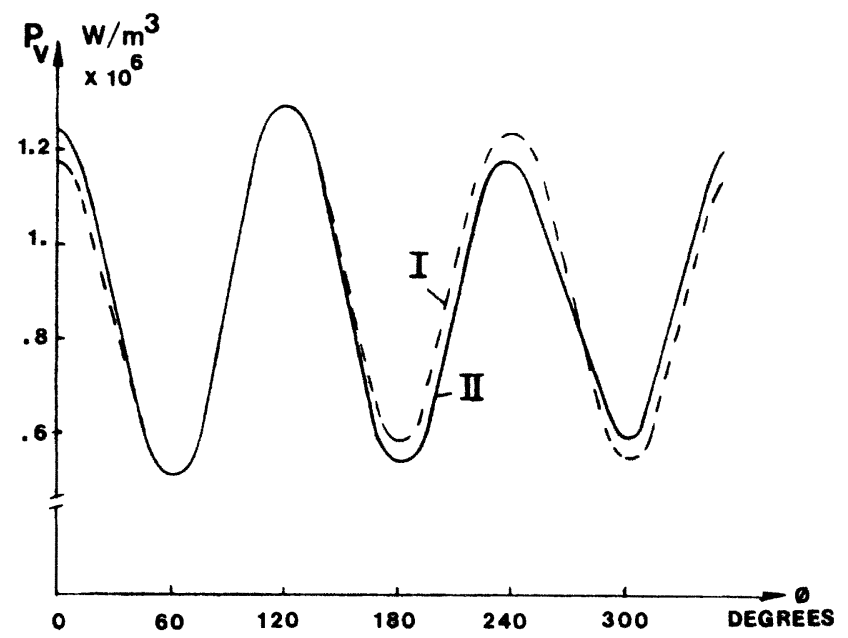

Fig. 6. Shell surface power loss density distribution for unbalanced electrode currents $A B C$ (I) and $C B A$ (II) sequence.

during furnace operation. Expressing this into a slight current unbalance (5 percent) and recalculating the power losses, curve $I$ in Fig. 6 is obtained. Reversing the phase sequence produces curve II.

It is interesting to observe the effect on the power loss density distribution caused by this current unbalance. As expected, the largest losses occur in the regions opposite the electrodes. However, compared to a balanced supply the ratio of maximum to minimum induced loss becomes larger, the distribution asymmetrical, and there is also a noticeable shift in the locations where the maxima occur. It can be shown that the corresponding electromagnetic force on the arc in a horizontal direction, hence, the direction of the arc flame to the refractory is similarly affected [3]. In addition, the resulting arc flames are changed, resulting in three different flare-out patterns. The effect of this is an underpowering of phase $A$ ( $A B C$ sequence), the so-called cold phase, or that of phase $C$, in the event where the polarities of electrodes $A$ and $C$ are reversed, see Fig. 7. The hot spot region under electrode $B$ (center phase), which is most severe, cannot be so influenced in this manner. 

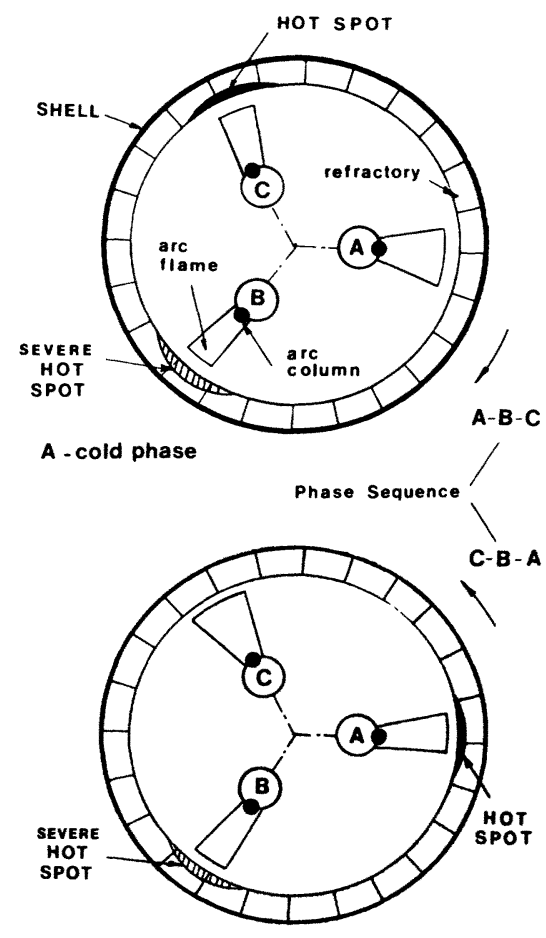

C - cold phase

Fig. 7. Dissimilar hot spots on refractory due to electrode current unbalances.

Knowing the power loss density throughout the furnace shell material, it is relatively simple to obtain the total electromagnetic torque acting on the shell or on the water-cooled panels. For this, the total power loss in the material must be calculated and from this the torque is obtained [6], namely

$$
T=\frac{P_{\text {total }}}{\omega} .
$$

Applying this to the furnace considered, the force acting along the circumference of the shell was calculated to be $120 \mathrm{~N}$ per unit length of panel. Even considering the largest size water cooling panel (with a length of approximately $3 \mathrm{~m}$ ), this force was not considered to be of any consequence in the installation. This was further substantiated by calculations on mechanical constraints and observations in the field on many installations.

\section{EXPERIMENTAL RESULTS-ARC FURNACE}

To illustrate the effect of the unequal arc flame patterns and directions, as well as to support the theoretical notation of the shifted hot spot on the refractory lining, Fig. 8 is included. It shows the measured refractory wear along the circumference of a section above the slag line [7]. It is evident that the wear pattern corresponds to that as predicted by the theory presented in this paper.

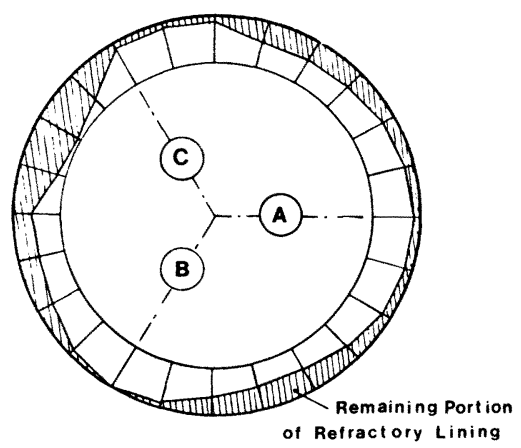

Fig. 8. Measured refractory wear pattern in cylindrical arc furnace shell.

\section{SUMMARY}

This article describes a method to calculate the eddy current losses and from those the mechanical forces acting on structural members (such as water-cooled panels), located along the circumference of an electric arc furnace shell. It also attempts to correlate some of the observed (and measured) refractory wear patterns with electromagnetic phenomena. Although the power loss distribution is not identical with the temperature distribution, there is a qualitative agreement, as shown. However, in relation to the arc heat radiation on the furnace side walls, the eddy current losses are relatively small. What is important is the corresponding thermal and electromagnetic phenomena and their seemingly interdependence. More work is being done to determine the electromagnetic forces acting on the arc column. As a result, it will be possible to predict the directionality and flare out of the arc flame with respect to the refractory wall. Results of this study will be reported at a later date.

\section{ACKNOWLEDGMENT}

The author gratefully acknowledges helpful discussions with V. Bulat, Chief Electrical Engineer at EMPCO (Canada) Ltd., during the preparation of this paper.

\section{REFERENCES}

[1] L. Voisinet, "New developments in arc melting," Modern Casting, Dec. 1977.

[2] W. E. Schwabe, "Fundamentals of heat distribution and refractory wear in electric steel furnaces," Iron and Steel Engineer, Dec. 1961.

[3] P. F. Ryff, "Electromagnetic forces on high current arcs," to be published.

[4] M. Stafl, Electrodynamics of Electrical Machines. Iliffe, 1967.

[5] Standard Mathematical Tables. Cleveland: CRC Press.

[6] J. Lammeraner and M. Stafl, Eddy Currents. Cleveland: CRC Press, 1964

[7] E. R. Wunsche, EMPCO (Canada) Ltd., company engineering report.

Peter F. Ryff (S'66-M ${ }^{\circ} 66-S^{\prime} 78$ ), for a photograph and biography, please see page 229 of this TRANSACTIONS. 